\title{
Modeling and propagation of near-field diffraction patterns: A more complete approach
}

\author{
Glen D. Gillen a) and Shekhar Guha \\ Air Force Research Laboratory, Materials and Manufacturing Directorate, WPAFB, Ohio 45433
}

(Received 5 December 2003; accepted 7 May 2004)

\begin{abstract}
We discuss the origins and regions of validity of various near-field diffraction models. The complete Rayleigh-Sommerfeld model is found to accurately represent intensity distributions for axial distances up to and including the location of the aperture, a region where commonly used models fail. We show that near-field diffraction theory can be applied to the refraction of light at an interface between two different media yielding results that demonstrate the validity of Snell's law in the presence of diffraction. Calculations using near-field diffraction and Fourier optics are compared to experimentally measured intensity distributions. (C) 2004 American Association of Physics Teachers.
\end{abstract}

[DOI: $10.1119 / 1.1767102]$

\section{INTRODUCTION}

Diffraction occurs when an aperture is illuminated by light. In spite of the historical importance of diffraction (the understanding of which first established the wave nature of light), a complete description of the distribution of light immediately beyond the illuminated aperture is not available in the usual textbooks of optics. ${ }^{1-6}$ The treatment of near-field diffraction in undergraduate textbooks ranges from simply stating the equations to be used for near-field and far-field diffraction regions, ${ }^{1}$ to briefly discussing the physical basis for the Fresnel and Fraunhofer diffraction models, ${ }^{2}$ to deriving Fresnel and Fraunhofer diffraction using scalar diffraction theory. ${ }^{3,4}$ For a more detailed discussion of scalar diffraction theory and derivations of models other than Fresnel and Fraunhofer, students are usually referred to graduatelevel textbooks. ${ }^{5,6}$

The simplest case of diffraction of light is that of plane waves traveling beyond a hard, planar aperture. The complexity of accurately modeling and predicting the electric field and intensity distributions at every point beyond the aperture can be computationally quite intensive. Frequently, various approximations are implemented to decrease the level of complexity and computational times required to model the diffracted beam propagation.

Over the past few decades, interest has slowly grown in reexamining near-field diffraction theory because of advancements in laser technology, the continual shrinking of technological components, and advancements in computer technology. One of the many recent advances in laser technology is the generation of terahertz radiation. ${ }^{7}$ The conversion of light wavelengths from the nanometer regime to the centimeter regime also results in a proportionate growth in the scale of near-field diffraction patterns, from the microscopic (sub-micron) scale to the macroscopic (millimeter and centimeter) scale. The presence of near-field optics on this macroscopic scale has fueled efforts to accurately characterize $\mathrm{e}^{7,8}$ and model near-field propagation of terahertz electromagnetic radiation. ${ }^{9}$

In addition to laser technology expanding the scales of near-field beam propagation, manufacturing technology continues to shrink the scale of optical components. Microelectrical-mechanical systems and optical micro-electricalmechanical components are now reaching the dimensions of tens ${ }^{10}$ to hundreds ${ }^{11}$ of microns. Modeling beam propagation within complex systems on this scale presents new challenges. ${ }^{12,13}$

The aim of this paper is to discuss various diffraction theory models, probe the regions of validity of each, and apply appropriate models to some specific examples. The discussion could be incorporated into an advanced undergraduate or beginning graduate level course in optics. We first derive and compare various models for near-field light propagation. Then we calculate some representative twodimensional intensity patterns and beam profiles for the nearfield diffraction of infrared laser light. In the first example we apply near-field diffraction theory to the traditional example of the refraction of light at an interface between two media. The locations and intensity profiles are discussed as functions of the incident angle of the light and the refractive index of the medium. Finally, we investigate the propagation of light beyond the aperture and through a lens, yielding some interesting results that are verified experimentally.

\section{THEORETICAL MODELING}

Studies of the propagation of light beyond an aperture were extensively performed in the late 19th century by Kirchhoff, ${ }^{14}$ Sommerfeld, ${ }^{15}$ and Rayleigh, ${ }^{16}$ among many others. Further progress in finding solutions that accurately describe the electric field distributions for all points beyond the aperture were hampered by the complexity of the integrals to be performed. Over the years, several different models have been developed for the electric field and intensity distributions for points beyond the aperture. The models make different assumptions and approximations to simplify the mathematics and reduce the computational time. Table I summarizes some of the models and the approximations used for each. As a result of the speed of present computer technology, numerical results for even the full wave equations can now be obtained using a desktop computer.

We begin by considering the situation depicted in Fig. 1 . Monochromatic plane waves of light travel in the positive $z$ direction and are incident upon an aperture in the $z=0$ plane. A volume of space after the $z=0$ plane is enclosed by two surfaces, $S_{0}$ and $S_{2} . S_{0}$ is planar in shape and lies in the $z$ $=0$ plane, and $S_{2}$ is an arbitrary surface in the $z>0$ region. The enclosed volume has a small hollow sphere with a surface of $S_{1}$. This sphere surrounds the point of interest, $P_{1}$. 
Table I. Various diffraction theory models and their approximations.

\begin{tabular}{ll}
\hline \hline Model & Approximation \\
\hline Full wave equations & None \\
Complete Rayleigh-Sommerfeld & Boundary conditions \\
on surfaces & $\rho \gg \lambda$ \\
Approximate Rayleigh-Sommerfeld & $\rho \gg \lambda$ \\
Fresnel-Kirchhoff & $z_{1}^{3} \gg \frac{\pi}{4 \lambda}\left[\left(x_{1}-x_{0}\right)^{2}+\left(y_{1}-y_{0}\right)^{2}\right]^{2}$ \\
Fresnel & $z_{1} \gg \frac{\pi}{\lambda}\left(x_{1}^{2}+y_{1}^{2}\right)$ \\
& \\
Fraunhofer & \\
\hline \hline
\end{tabular}

We use the divergence form of Gauss' theorem, several vector identities, and a particular choice of a vector function to arrive at Green's scalar theorem, ${ }^{6}$

$$
\begin{aligned}
& \iint_{S}(U \vec{\nabla} V-V \vec{\nabla} U) \cdot \hat{n} d s \\
& \quad=\iiint_{\nu}\left(U \nabla^{2} V-V \nabla^{2} U\right) d v,
\end{aligned}
$$

where $U$ and $V$ are arbitrary scalar fields that are functions of position. The only restriction on the functions $U$ and $V$ are that they be smooth and continuous at all points in space. The surface integration is over all surfaces: $S_{0}, S_{1}$, and $S_{2}$. The volume integration is over the volume enclosed by $S_{0}$ and $S_{2}$, with the exception of the volume enclosed by $S_{1}$. If $U$ and $V$ also are restricted to be solutions of the Helmholtz wave equation,

$$
\left(\nabla^{2}+k^{2}\right) U=0 \text { and }\left(\nabla^{2}+k^{2}\right) V=0,
$$

then the volume integral is equal to zero, and Eq. (1) becomes

$$
\begin{aligned}
& \iint_{S_{0}}(U \vec{\nabla} V-V \vec{\nabla} U) \cdot \hat{n} d s_{0} \\
& \quad+\iint_{S_{2}}(U \vec{\nabla} V-V \vec{\nabla} U) \cdot \hat{n} d s_{2} \\
& +\iint_{S_{1}}(U \vec{\nabla} V-V \vec{\nabla} U) \cdot \hat{n} d s_{1}=0 .
\end{aligned}
$$

If we let $V$ represent a component of the electric field, and the radius of $S_{1}$ go to zero, it can be shown (see Ref. 6, p. 42) that the integral over $S_{1}$ becomes

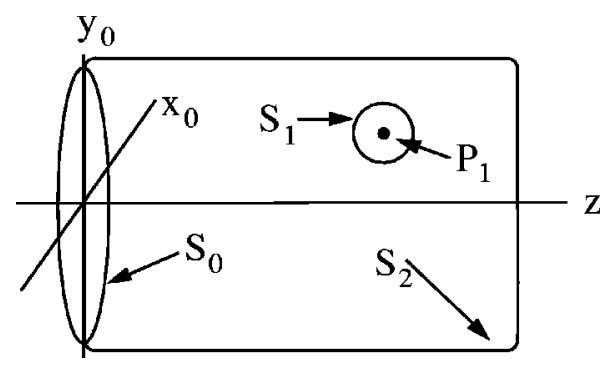

Fig. 1. Representation of volumes and surfaces used in establishing a diffraction theory.

$$
\iint_{S_{1}}\left(U \vec{\nabla} E_{i}-E_{i} \vec{\nabla} U\right) \cdot \hat{n} d s_{1}=4 \pi E_{i}\left(P_{1}\right),
$$

where $E_{i}$ represents either the $x, y$, or $z$ component of the electric field for each point on the surface, and $E_{i}\left(P_{1}\right)$ is the $i$ th component at position $P_{1}$. If we use the Sommerfeld radiation condition ${ }^{6}$ that the radiation across the surface $S_{2}$ is composed of only outward waves and let the distance from $P_{1}$ to the surface of $S_{2}$ become very large, the integral over $S_{2}$ also vanishes. Equation (3) now becomes

$$
E_{i}\left(P_{1}\right)=\frac{1}{4 \pi} \iint_{S_{0}}\left(U \vec{\nabla} E_{i}-E_{i} \vec{\nabla} U\right) \cdot \hat{n} d s_{0} .
$$

Rayleigh-Sommerfeld boundary conditions on the aperture plane state that in the aperture region of the aperture plane the field distribution $U$ and its normal derivative are exactly as they would be in the absence of the screen; everywhere else in the aperture plane either the field distribution $U$ or its normal derivative is exactly zero. Both of these boundary conditions on $U$ in the aperture plane are satisfied by the following Green's function:

$$
U=\frac{e^{-i k\left|\vec{r}_{2}-\vec{r}_{0}\right|}}{\left|\vec{r}_{2}-\vec{r}_{0}\right|}-\frac{e^{-i k\left|\vec{r}_{1}-\vec{r}_{0}\right|}}{\left|\vec{r}_{1}-\vec{r}_{0}\right|}
$$

where $\vec{r}_{0}$ denotes the vector from the origin to a point on the $z=0$ plane, $\vec{r}_{1}$ denotes the vector from the origin to point $P_{1}$, and $\vec{r}_{2}$ denotes a mirror image of point $P_{1}$ on the negative side of the $z=0$ plane. The scalar function $U$ in Eq. (6) satisfies the Rayleigh-Sommerfeld boundary conditions and represents a point source located at $\vec{r}_{1}$ and a mirror image point source located at $\vec{r}_{2}$ oscillating exactly $180^{\circ}$ out of phase with each other. If we substitute Eq. (6) into Eq. (7), it can be shown that the field at $P_{1}$ is

$$
\vec{E}\left(P_{1}\right)=\frac{k z_{1}}{i 2 \pi} \iint E_{z=0} \frac{e^{i k \rho}}{\rho^{2}}\left(1-\frac{1}{i k \rho}\right) d x_{0} d y_{0},
$$

where

$$
\rho=\sqrt{\left(x_{1}-x_{0}\right)^{2}+\left(y_{1}-y_{0}\right)^{2}+z_{1}^{2}} .
$$

Equation (7) is the full Rayleigh-Sommerfeld solution. The only assumptions are the boundary conditions on the surfaces $S_{0}$ and $S_{2}$. The Rayleigh-Sommerfeld diffraction integral is more commonly expressed in its simplified form as

$$
\vec{E}\left(P_{1}\right)=\frac{k z_{1}}{i 2 \pi} \iint \vec{E}_{z=0} \frac{e^{i k \rho}}{\rho^{2}} d x_{0} d y_{0},
$$

where it has been assumed that $\rho \gg \lambda .{ }^{6}$ Equation (9) is also known as the Fresnel-Kirchhoff diffraction integral.

If Eq. (8) is expanded and we assume that

$$
z_{1}^{3} \gg \frac{\pi}{4 \lambda}\left[\left(x_{1}-x_{0}\right)^{2}+\left(y_{1}-y_{0}\right)^{2}\right]^{2},
$$

and $\rho^{2} \approx z^{2}$ in the denominator of Eq. (9), we arrive at the Fresnel (paraxial) near-field diffraction integral, 


$$
\begin{aligned}
\vec{E}_{1}\left(P_{1}\right)= & \frac{k e^{i k z_{1}}}{i 2 \pi z_{1}} \\
& \times \iint \vec{E}_{z=0} e^{\left(i k / 2 z_{1}\right)\left(\left(x_{1}-x_{0}\right)^{2}+\left(y_{1}-y_{0}\right)^{2}\right)} d x_{0} d y_{0} .
\end{aligned}
$$

Equation (11) can be expressed in cylindrical coordinates for a circular aperture of radius $a$ as

$$
\begin{aligned}
\vec{E}\left(P_{1}\right)= & \frac{2 \pi e^{i k z_{1}}}{i k z_{1}} \\
& \times e^{-i k r_{1}^{2} / 2 z_{1}} \int_{0}^{a} \vec{E}_{z=0} e^{-i k r_{0}^{2} / 2 z_{1}} J_{0}\left(\frac{k r_{0} r_{1}}{z_{1}}\right) r_{0} d r_{0},
\end{aligned}
$$

where $r_{0}$ and $r_{1}$ are the radial coordinates in the planes $z$ $=0$ and $z=z$, respectively, and the point $P_{1}$ is $\left(x_{1}, y_{1}, z\right)$.

\section{CALCULATED RESULTS}

Figure 2 illustrates the on-axis calculated intensities for the three models discussed thus far. The integration of the diffraction integral for each model derived in Sec. II was done using Mathcad. Figure 2(a) is the calculated on-axis intensity using the complete Rayleigh-Sommerfeld model, Eq. (7), and Fig. 2(b) is calculated using the approximate Rayleigh-Sommerfeld or Fresnel-Kirchhoff model, Eq. (9); Fig. 2(c) is calculated using Fresnel's paraxial approximation, Eq. (11). Each graph of Fig. 2 is calculated as a function of position along the $z$-axis with $x_{1}=y_{1}=0$, for incident plane waves with a wavelength of $10 \mu \mathrm{m}$ and a round aperture with a radius of $100 \mu \mathrm{m}$ in the aperture plane. The amplitude of the electric field of the incident light is assumed to be unity.

According to Fig. 2(a), if we were to place a detector at position $(0,0, z)$, where $z$ is on the order of $1000 a$, or 1000 aperture radii, and observe the central intensity as we reduce $z$, we would first observe an increasing intensity as a function of smaller distances to the aperture, which is what we would expect. But, the increasing axial intensity does not simply continue to increase all the way to the location of the aperture, as would be expected for a $1 / z^{2}$ intensity dependence for a point source. Instead, a primary maximum is reached at a position of $10 a$ and an intensity oscillation is observed for smaller values of $z$, with an overall amplitude decrease. It also would be expected that as $z$ asymptotically approaches the position of the aperture, the intensity should approach that of the incident plane waves, or unity for this example. Closer inspection of Fig. 2(a) reveals that the observed number of on-axis maxima is equal to $a / \lambda$ (which also is observed for other calculations performed using a variety of $a / \lambda$ values). For optically dense materials, $n>1$, the longitudinal location of the diffraction pattern also is proportional to $n$ beyond the aperture, and will be discussed in Sec. IV.

The vertical arrows in Figs. 2(b) and 2(c) represent the lower region of validity of each model, as defined in Table I. For Fig. 2(b), the vertical arrow is at $z=\lambda$, while the arrow in Fig. 2(c) is at $z=\pi \rho^{4 / 3} / 4 \lambda$. Figure 3 is a closer view of the longitudinal region where the approximate models begin to deviate from the axial intensity distributions calculated using
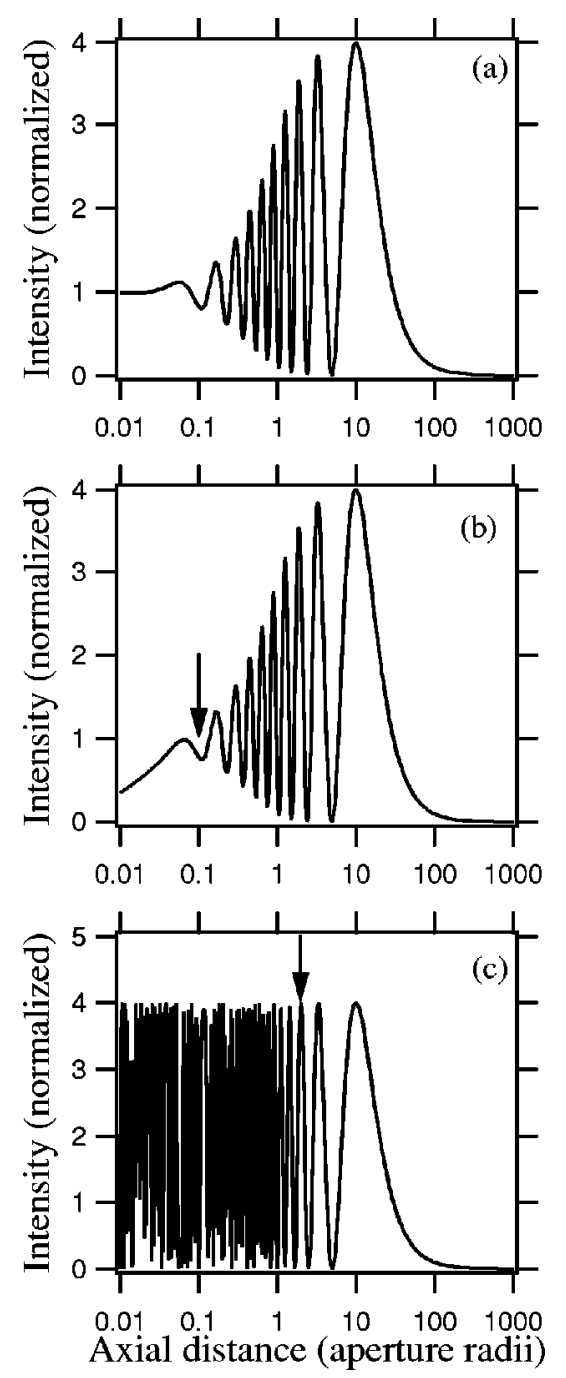

Fig. 2. Calculated on-axis intensity distributions for (a) the complete Rayleigh-Sommerfeld, (b) approximate Rayleigh-Sommerfeld, and (c) the Fresnel diffraction model. In (b) and (c) the arrow represents the lower limit of the validity of the model.

the complete Rayleigh-Sommerfeld model. The vertical arrows in Figs. 3(a) and 3(b) are at the same longitudinal distance as those in Figs. 2(b) and 2(c). As would be expected, the deviation of each approximate model is gradual; starting slightly beyond the lower validity threshold and deviating farther and farther from the complete model as the aperture is approached. The difference between the complete RayleighSommerfeld model, Eq. (7), and the approximate model, Eq. (9), is primarily an amplitude inaccuracy, whereas the paraxial approximation, Eq. (12), has both amplitude and phase inaccuracies.

In the same spirit as Fig. 2, Fig. 4 is an image plot as a function of the distance from the aperture, $z$, and the radial distance from the $z$-axis, using the complete RayleighSommerfeld model and the same aperture and laser field as in Fig. 2. The integration of Eq. (7) is repeated for every point in a grid in the $y-z$ plane. If a screen were to be placed at a particular distance from the aperture, then the observed image would be radially symmetric with a radial intensity profile equal to a vertical stripe through Fig. 4. To help illustrate the intensity profiles and radial symmetries, Fig. 5 shows a collection of radial intensity distributions for various 

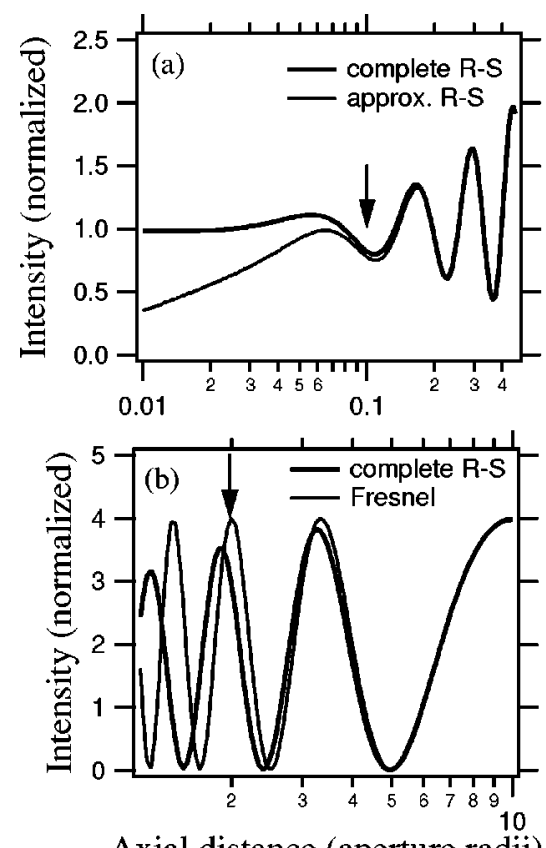

Axial distance (aperture radii)

Fig. 3. Comparison of the complete Rayleigh-Sommerfeld model to the (a) approximate Rayleigh-Sommerfeld and the (b) Fresnel models.

distances from the aperture as calculated directly from Eq. (7), for points along the $(0, y, z)$ line where $z$ is held constant.

The four beam profiles of Fig. 5 are a demonstration of the variety of radial intensity distributions that can be observed as a function of distance from the aperture. Figure 5(a) is calculated for a distance of 10 aperture radii, $1 \mathrm{~mm}$, or the location of the primary maxima. A single spot would be observed on an image screen placed at this location with the intensity at the center four times greater than that of the incident light on the aperture plane. Figure 5(b) is calculated for a distance of 4.9 aperture radii, $490 \mu \mathrm{m}$, or the location of the first minima. If an image screen were to be placed there, a single "doughnut" shape would be observed. Figure 5(c) shows the calculated radial intensity distribution for a distance of 1.86 aperture radii, or the location of the third maxima. At this location, a bright central spot would be observed circumscribed by two blurry circles of light. As the image screen is moved closer to the aperture, the central region oscillates between a maximum and a minimum and the number of rings surrounding it increases in both number

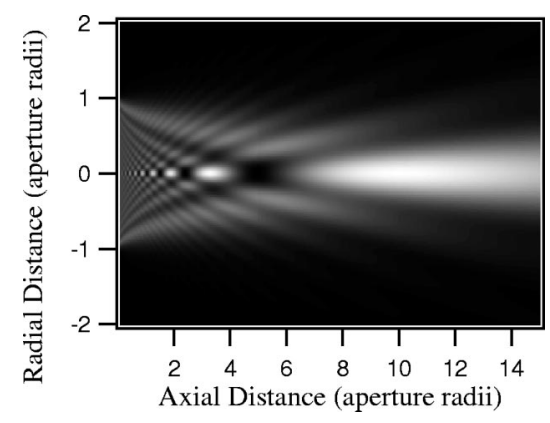

Fig. 4. Calculated intensity distributions as a function of both radial and axial distance from the aperture using the complete Rayleigh-Sommerfeld model. White represents the maximum intensity, black the minimum intensity, and grey in between.
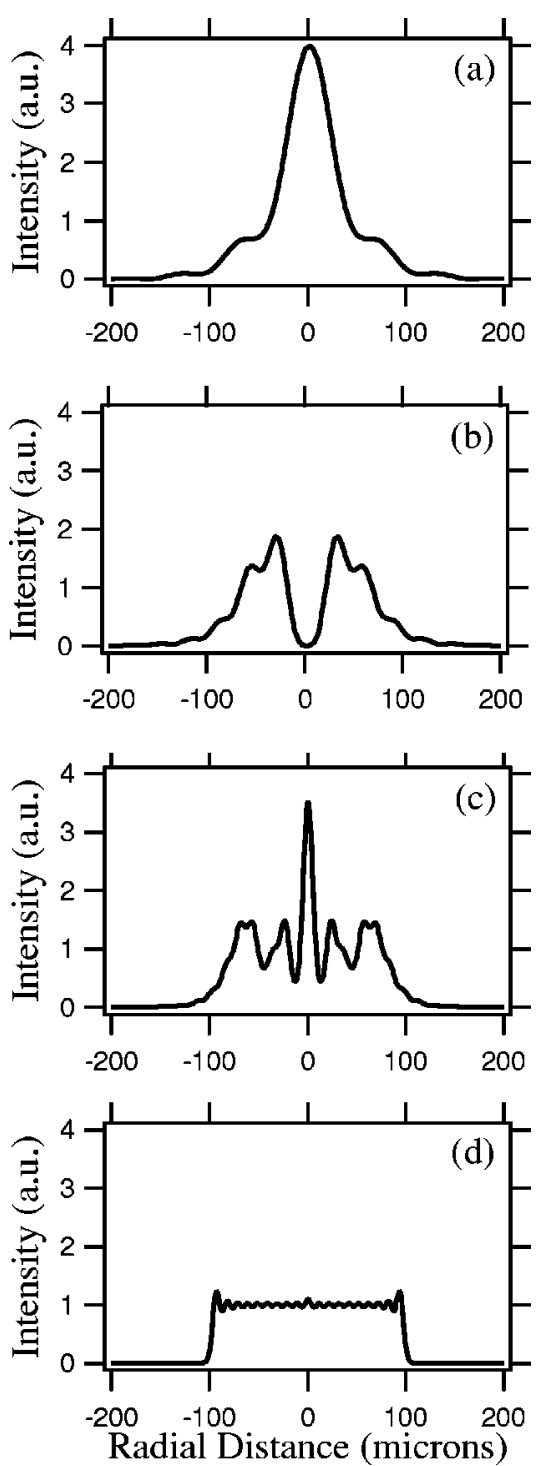

Fig. 5. Calculated radial intensity beam profiles for axial distances of (a) 10 , (b) 4.9 , (c) 1.86 , and (d) 0.05 multiples of the aperture radii. The vertical axes are normalized by an incident intensity on the aperture of unity.

and size, eventually fading together (as the contrast between the maxima and minima decreases) to form a near uniform spot with a radius equal to that of the aperture and an intensity of unity, as seen in Fig. 5(d). The latter represents a calculation for a distance of only $5 \mu \mathrm{m}$ from a $200 \mu \mathrm{m}$ diameter aperture.

\section{VERIFICATION OF SNELL'S LAW USING NEAR-FIELD DIFFRACTION}

We next apply the complete Rayleigh-Sommerfeld diffraction model to the refraction of light at the interface of two different media and compare the results to those predicted using Snell's law of refraction and geometrical optics. For plane waves incident upon the aperture at an angle other than normal, a position-dependent phase term must be included at the aperture plane. The electric field of the incident light can be written as

$$
\vec{E}\left(x_{0}, y_{0}, 0\right)=e^{i 2 \pi n_{0} x_{0} \sin (\theta) / \lambda} \hat{x},
$$




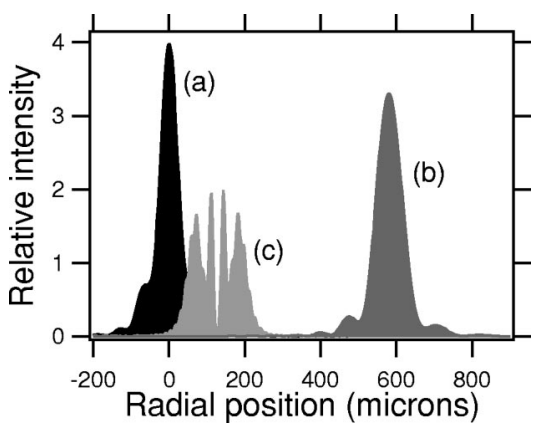

Fig. 6. Radial intensity profiles for plane waves with a wavelength of 10 $\mu \mathrm{m}$, incident upon a $200 \mu \mathrm{m}$ diameter aperture, and an aperture-image plane distance of $1 \mathrm{~mm}$ for (a) $n=n_{0}=1, \theta=0^{\circ}$, (b) $n=n_{0}=1, \theta=30^{\circ}$, and (c) $n=4, n_{0}=1, \theta=30^{\circ}$.

where $n_{0}$ is the refractive index of in the medium before the aperture, $x_{0}$ is the radial distance from the center of the aperture in the $x$ direction, $\theta$ is the angle of incidence, and $\hat{x}$ is the polarization direction of the laser light. The amplitude of the electric field is again assumed to be unity. If we substitute Eq. (13) into Eq. (7) and use $n$ as the refractive index of the medium after the aperture, we obtain

$$
\begin{aligned}
\vec{E}\left(P_{1}\right)= & \frac{n z_{1}}{i \lambda} \iint e^{i 2 \pi n_{0} x_{0} \sin (\theta) / \lambda} \\
& \times \frac{e^{i 2 \pi n \rho / \lambda}}{\rho^{2}}\left(1-\frac{\lambda}{i 2 \pi n \rho}\right) d x_{0} d y_{0} \hat{x} .
\end{aligned}
$$

Equation (15) is integrated over all points in the aperture plane for each point on the image plane. The integral is then repeated for points along the line $\left(x, 0, z_{p}\right)$, where $z_{p}$ is constant, and is the distance from the aperture to the longitudinal position of the image plane of interest. The beam intensity profiles as calculated are a function of $x$ using this method and are displayed in Fig. 6. The experimental conditions for Fig. 6 are those of a collimated $\mathrm{CO}_{2}$ laser, with a wavelength of $10 \mu \mathrm{m}$, incident upon an aperture with a radius of 100 $\mu \mathrm{m}$. The beam waist is assumed to be much larger than the aperture (so a constant field amplitude across the aperture can be assumed). The distance from the aperture to the image plane is chosen to coincide with the primary maxima of the interference pattern for beam propagation through air or vacuum after the aperture.

We first calculate the beam profile using Eq. (14) for light waves normally incident upon the aperture and for a refractive index of the medium after the aperture equal to that before the aperture plane, $n=n_{0}=1$. The resulting beam profile is shown in Fig. 6(a) and is centered at $x=0$. This beam intensity profile is the same as that of Fig. 4(a) which was calculated using Eq. (7) for the same experimental conditions. If we use geometrical optics and Snell's law, the expected intensity profile would be a perfect copy of the uniform "flat-top" intensity profile incident upon the aperture and centered around $x=0$, that is, a beam profile having a constant intensity with amplitude of unity would be expected from $-100 \mu \mathrm{m}$ to $+100 \mu \mathrm{m}$ with sharp edges. Figure 6(a) shows that if we incorporate the wave nature of light and a mathematically rigorous model of diffraction theory, we observe a much different intensity profile.

If we perform a similar calculation, except allow for an angle of incidence of $30^{\circ}$, we might expect a similar beam profile offset from $x=0^{\circ}$ on the image plane by $z \tan (\theta)$, or $577 \mu \mathrm{m}$ at $z=1 \mathrm{~mm}$. The resulting intensity profile is illustrated in Fig. 6(b) and is centered on $x=578 \mu \mathrm{m}$. The difference between the transverse locations of the two is within a step size $(30 \mu \mathrm{m})$ of the Rayleigh-Sommerfeld calculation. The geometrical shadow of the $200 \mu \mathrm{m}$ diameter pinhole for light incident at an angle of $30^{\circ}$ would be an ellipse with a minor diameter of $200 \mu \mathrm{m}$ in the $y$ dimension, and a major diameter of $231 \mu \mathrm{m}$ in the $x$ dimension. The uniform intensity would have a value of 0.866 for an incident electric field amplitude of unity. This value is slightly higher than the ratio of the peak heights of Figs. 6(a) and 6(b) of 0.825. But, neither of these intensity profiles of Fig. 6 is close to uniform, as predicted using geometrical optics. Closer inspection of Fig. 6(b) reveals that the intensity distribution is not just simply wider and lower than that of Fig. 6(a), as would be expected using geometrical optics, but actually has some asymmetries in the outer-lying rings; another result of the wave nature of light using diffraction theory.

Finally, to truly compare the results of RayleighSommerfeld calculations to Snell's law, we include an optically dense medium in the region after the aperture. The refractive index chosen for this calculation is that of germanium, a common optical material for long-wave infrared applications, with a linear refractive index of $n=4$. For each point on the $x$-axis, and a distance $z=1 \mu \mathrm{m}$ away, Eq. (14) is integrated using $n_{0}=1, n=4$, and $\theta=30^{\circ}$. The resulting intensity profile is illustrated in Fig. 6(c). If we use Snell's law of refraction, the image would be centered on $x=126 \mu \mathrm{m}$, which is the precise location of the minimum in the middle of Fig. 6(c).

The greatest difference between Figs. 6(b) and 6(c), other than their locations on the $x$-axis, is the difference in their intensity profiles. As the refractive index of the medium after the aperture changes, so does the relative distance to a particular interference pattern. For example, the intensity profile calculated for Fig. 6(b) is very similar to the beam profile calculated for $n=1, \theta=0^{\circ}$ and a longitudinal distance closer to the aperture by a factor of 4 (the $n$ value of germanium). The net result of beam propagation through a nonabsorbing, optically dense medium after the aperture is a diffraction pattern similar to that of Fig. 4, except the longitudinal axis is increased by a factor of $n$.

\section{PROPAGATION OF NEAR-FIELD PATTERNS THROUGH A LENS}

Modeling light propagation through a hard aperture is just one step in accurately modeling beam propagation through a complex optical train of components. The addition of a lens to the system is an example of applying near-field diffraction theory to more complex systems. The lens is placed after the aperture so that the entire optical "train" for this example consists of a circular hard aperture and a lens some distance beyond the aperture.

The general approach for modeling this example is depicted in Fig. 7. The incident light on the aperture, located in plane $1(z=0)$, is assumed to be a plane wave traveling in the $+z$ direction. The electric field for every point in plane 2 is calculated using a suitable near-field diffraction theory. The electric field is then calculated for plane 3 by incorporating the changes to the electric field induced by the lens. Finally, by using near-field diffraction theory, the electric 


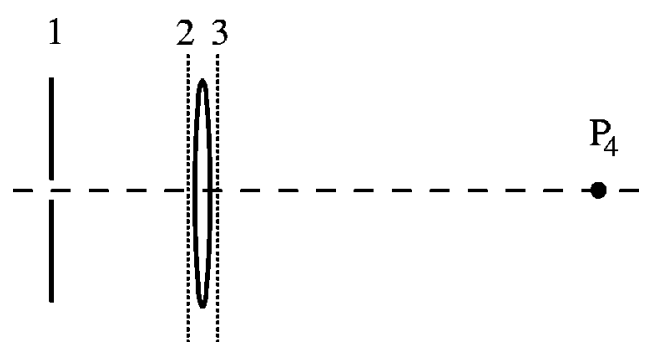

Fig. 7. Experimental setup for the propagation of near-field diffraction patterns through a lens. The $5 \mathrm{in}$. focal length lens is placed $6.25 \mathrm{in}$. away from a $2 \mathrm{~mm}$ diameter pinhole. The incident light is from a $10.6 \mu \mathrm{m}$ wavelength, $\mathrm{CO}_{2}$ laser.

field at the point of interest, $P_{4}$, is calculated by propagating the electric field in plane 3 to the point of interest.

The near-field diffraction model chosen to calculate the electric field distribution on plane 2, just before the lens, is that of Fresnel diffraction, or Eq. (12). For this example, the paraxial approximation was chosen over the complete Rayleigh-Sommerfeld model because the axial distance between the aperture and the lens is well within the region of validity of this approximation. If we use Eq. (12), we can express the electric field everywhere on plane 2 as

$$
\begin{aligned}
\vec{E}_{2}\left(r_{2}, z_{2}\right)= & \frac{2 \pi e^{i k z_{2}}}{i k z_{2}} e^{-i k r_{2}^{2} / 2 z_{2}} \\
& \times \int_{0}^{a} \vec{E}\left(r_{1}\right) e^{-i k r_{1}^{2} / 2 z_{2}} J_{0}\left(\frac{k r_{1} r_{2}}{z_{2}}\right) r_{1} d r_{1} .
\end{aligned}
$$

As the mathematical model for the lens, the Fourier optical thin lens approximation was chosen. ${ }^{6}$ Thus, planes 2 and 3 are coplanar, and the electric field at plane 3 is the electric field at plane 2 multiplied by a quadratic phase transformation term,

$$
E_{3}\left(r_{3}, z\right)=E_{2} e^{-i(k / 2 f) r_{3}^{2}} e^{-i k n_{1} \Delta_{0}},
$$

where $f$ is the focal length of the lens, $k$ is the wave number of the light, $n_{1}$ is the refractive index of the lens material, and $\Delta_{0}$ is the lens thickness at the center.

The final step in this example is to calculate the electric field for points of interest beyond the lens. Once again, we are interested only in the electric field at points far from the axial position of the lens and choose to use the paraxial approximation using plane 3 as the aperture plane and Eq. (16) as the input electric field. The electric field at point $P_{4}$ is

$$
\vec{E}\left(P_{4}\right)=\frac{2 \pi e^{i k z_{4}}}{i k z_{4}} \int_{0}^{\infty} \vec{E}_{3}\left(r_{3}\right) e^{-i k r_{3}^{2} / 2 z_{4}} J_{0}\left(\frac{k r_{3} r_{4}}{z_{4}}\right) r_{3} d r_{3} .
$$

The substitution of Eq. (15) into Eq. (16), and Eq. (16) into Eq. (17), leads to a complicated function. The quadratic exponential and Bessel function terms of the infinite integral of the resulting equation can be reduced to a quadratic exponential term and a modified Bessel function, eliminating the infinite integral. ${ }^{17}$ Further simplifications lead to the following expression for the electric field for any point $P_{4}\left(r_{4}, z_{4}\right)$ :

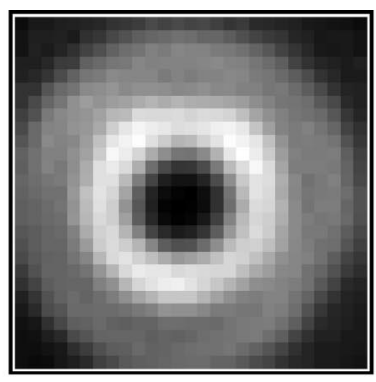

(a)

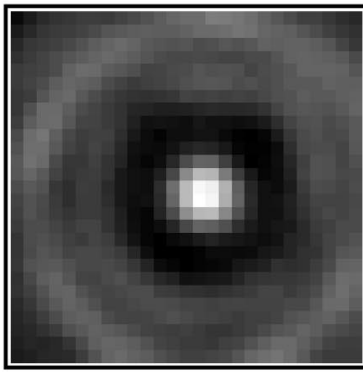

(b)

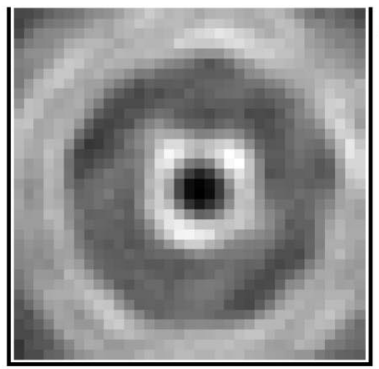

(c)

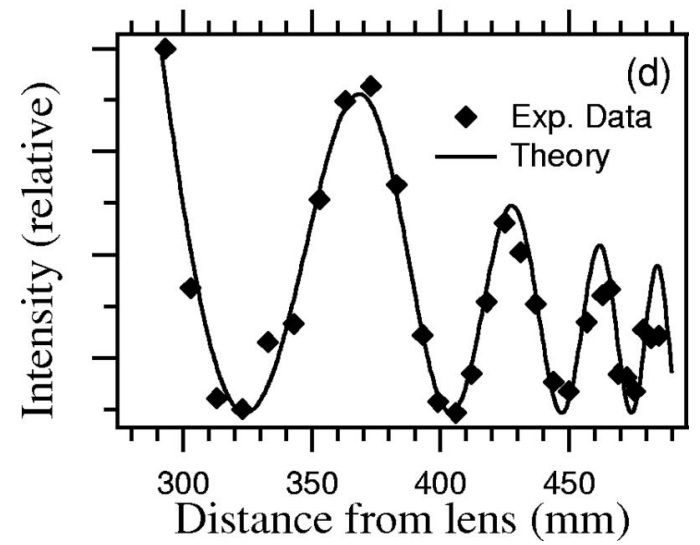

Fig. 8. Beam intensity profiles measured over distances of (a) $313 \mathrm{~mm}$, (b) $373 \mathrm{~mm}$, and (c) $406 \mathrm{~mm}$ from the lens. The image scans for (a) and (b) cover an area of $2 \mathrm{~mm}$ by $2 \mathrm{~mm}$, while the image for (c) is an area of 2.625 $\mathrm{mm}$ by $2.625 \mathrm{~mm}$. (a) The experimental and calculated on-axis relative intensities versus axial distance from the lens.

$$
\begin{aligned}
E_{4}\left(r_{4}, z_{4}\right)= & \frac{i d_{1}}{d_{21} d_{42}} \exp \left[-i \frac{k r_{41}^{2}}{2 d_{42}}\left(1-\frac{d_{1}}{d_{42}}\right)\right] \\
& \times \int_{0}^{1} \exp \left[-i \frac{k r_{11}^{2}}{2 d_{21}}\left(1-\frac{d_{1}}{d_{21}}\right)\right] \\
& \times J_{0}\left(\frac{d_{1} r_{11} r_{41}}{d_{21} d_{42}}\right) r_{11} d r_{11},
\end{aligned}
$$

where $r_{11}, r_{41}, d_{21}, d_{42}$, and $d_{1}$ are the dimensionless quantities: 


$$
\begin{aligned}
& r_{11}=\frac{r_{1}}{a}, \quad r_{41}=\frac{r_{4}}{a}, \\
& d_{21}=\frac{z_{2} \lambda}{2 \pi a^{2}}, \quad d_{42}=\frac{\left(z_{4}-z_{2}\right) \lambda}{2 \pi a^{2}}, \\
& d_{1}=\frac{1}{d_{21}}+\frac{1}{d_{42}}-\frac{1}{f} .
\end{aligned}
$$

The distance $a$ is the aperture radius.

For this example, the experimental conditions chosen are those of $\mathrm{CO}_{2}$ laser light (10.6 $\mu \mathrm{m}$ wavelength) incident upon a $2 \mathrm{~mm}$ diameter circular aperture and passing through a 5 in. focal length lens placed $6.25 \mathrm{in}$. beyond the aperture. This particular arrangement is chosen using hindsight from a previous experimental setup that produced large variations in the transverse beam profiles as a function of the longitudinal distance from the lens. Figures 8(a)-8(c) are examples of beam profiles measured for a variety of axial distances from the lens. The beam profiles of Figs. 8(a) and 8(b) span a space of $2 \mathrm{~mm}$ by $2 \mathrm{~mm}$, and the beam image of Fig. 8(c) spans $2.625 \mathrm{~mm}$ by $2.625 \mathrm{~mm}$. As we look at the beam profile just beyond the lens and slowly move farther away, the observed image is initially a large Gaussian-style spot that grows in central intensity and narrows in width. After reaching a maximum intensity and minimum beam waist, the beam profile does not exhibit typical Gaussian characteristics. Instead, the beam profile characteristics match those of beam profiles of near-field diffraction patterns, calculated in Figs. 4 and 5. Figure 8(a) shows a beam intensity scan for a distance of $313 \mathrm{~mm}$ from the lens where a single radial minimum is observed in the center, similar to the first minimum observed as an illuminated aperture in the near-field regime is approached [see Fig. 5(b)]. Figures 8(b) and 8(c) are beam intensity scans for axial locations of the next maxima and minima observed for increasing $z$. For each beam intensity scan measured, the relative central intensity is displayed in Fig. 8(d) as a function of the distance from the lens. The solid line displayed in Fig. 8(d) is a result of numerically integrating Eq. (18) for points along the axis of propagation for the same experimental conditions. Calculated on-axis intensities predict a single bright spot followed by oscillations with an overall decreasing amplitude. The agreement between the observed relative on-axis intensities and those predicted using near-field diffraction is consistent out to a distance of approximately $470 \mathrm{~mm}$. Beyond this distance, the maxima and minima of the experimental beam scans begin to blur together as the contrast between them decreases.

\section{CONCLUSIONS}

We have used Gauss' theorem, several vector identities, and Green's scalar theorem to derive the complete and approximate Rayleigh-Sommerfeld diffraction integrals and the Fresnel (or paraxial) diffraction integral, and have dis- cussed the approximations and limitations of each. The complete Rayleigh-Sommerfeld model is applied to the traditional geometrical optics example of refraction of light at an interface between two different optical media. The results of the calculation not only agree with predictions using geometrical optics, but also demonstrate the wave nature of light and the diffraction behavior of physical optics. We also demonstrated that electromagnetic wave propagation through more complex optical systems can be modeled using nearfield diffraction techniques. The propagation of near-field diffraction patterns through a simple optical system is demonstrated as they manifest themselves farther down the optical train, and are magnified from the sub-millimeter to the decimeter scale. The observed on-axis intensity variations are consistent with calculations using Fresnel diffraction integrals and Fourier optics.

a)Electronic address: ggillen@anteon.com; also at Anteon Corporation, Dayton, Ohio 45433.

${ }^{1}$ Frank L. Pedrotti and Leno S. Pedrotti, Introduction to Optics (PrenticeHall, Englewood Cliffs, NJ, 1987).

${ }^{2}$ Francis A. Jenkins and Harvey E. White, Fundamentals of Optics (McGraw-Hill, New York, NY 1976), 4th ed.

${ }^{3}$ Grant R. Fowles, Introduction to Modern Optics (Dover, New York, 1975), 2nd ed.

${ }^{4}$ Eugene Hecht, Optics (Addison-Wesley, San Francisco, CA, 2002), 4th ed.

${ }^{5}$ Max Born and Emil Wolf, Principles of Optics (Cambridge U.P., New York, 1999), 7th ed.

${ }^{6}$ Joseph W. Goodman, Introduction to Fourier Optics (McGraw-Hill, New York, 1996), 2nd ed.

${ }^{7}$ B. I. Greene, J. F. Federici, D. R. Dykaar, R. R. Jones, and P. H. Bucksbaum, "Intererometric characterization of 160 fs far-infrared light pulses," Appl. Phys. Lett. 59, 893-895 (1991).

${ }^{8}$ R. R. Jones, D. You, and P. H. Bucksbaum, "Ionization of Rydberg atoms by subpicosecond half-cycle electromagnetic pulses," Phys. Rev. Lett. 70, 1236-1239 (1993).

${ }^{9}$ E. Budiarto, N.-W. Pu, S. Jeong, and J. Bokor, "Near-field propagation of terahertz pulses from a large-aperture antenna," Opt. Lett. 23, 213-215 (1997).

${ }^{10}$ D. M. Bloom, "Grating light valve: revolutionizing display technology," Proc. SPIE 3013, 165-171 (1997).

${ }^{11}$ L. Y. Lin, E. L. Goldstein, and R. W. Tkach, "Free-space micromachined optical switches with submillisecond switching time for large-scale optical crossconnects," IEEE Photonics Technol. Lett. 10, 525-527 (1998).

${ }^{12}$ T. P. Kurzweg, "Optical propagation methods for system-level modeling of optical MEM systems," Ph.D. thesis, Electrical Engineering, University of Pittsburgh, PA, 2002.

${ }^{13}$ S. P. Levitan, J. A. Martinez, T. P. Kurzweg, A. J. Davare, M. Kahrs, M. Bails, and D. M. Chiarulli, "System simulation of mixed-signal multidomain microsystems with piecewise linear models," IEEE Trans. Comput.-Aided Des. 22, 139-154 (2003).

${ }^{14}$ G. R. Kirchhoff, "Zur theorie der lichtstrahlen," Ann. Phys. (Leipzig) 18, 663-695 (1883).

${ }^{15}$ A. Sommerfeld, "Zur mathematischen theorie der beugungsercheinungen,” Nachr. Kgl. Akad. Wiss. Göttingen 4, 338-342 (1894)

${ }^{16}$ Lord Rayleigh, "On the passage of waves through apertures in plane screens, and allied problems," Philos. Mag. 43, 259-272 (1897).

${ }^{17}$ I. S. Gradshteyn and I. M. Ryzhik, Table of Integrals, Series, and Products (Academic, New York, 1980), 4th ed., Eq. (6.633.2.). 